[2] X. Geng and J. Hammer, "Input/output control of asynchronous sequential machines," IEEE Trans. Autom. Control, vol. 50, no. 12, pp. 1956-1970, Dec. 2005.

[3] N. Venkatraman and J. Hammer, "On the control of asynchronous sequential machines with infinite cycles," Int. J. Control, vol. 79, no. 7, pp. 764-785, 2006.

[4] M. D. Di Benedetto, A. Sangiovanni-Vincentelli, and T. Villa, "Model matching for finite-state machines," IEEE Trans. Autom. Control, vol. 46, no. 11, pp. 1726-1743, Nov. 2001.

[5] J.-M. Yang and J. Hammer, "State feedback control of asynchronous sequential machines with adversarial inputs," Int. J. Control, vol. 81, no. 12, pp. 1910-1929, 2008.

[6] Z. Kohavi, Switching and Finite Automata Theory, 2nd ed. New York: McGraw-Hill, 1978.

[7] A. Tiwari and K. A. Tomko, "Enhanced reliability of finite-state machines in FPGA through efficient fault detection and correction," IEEE Trans. Reliab., vol. 54, no. 3, pp. 459-467, Sep. 2005.

\section{Input and Output Quantized Feedback Linear Systems}

Daniel F. Coutinho, Minyue Fu, and Carlos E. de Souza

\begin{abstract}
Although there has been a lot of research on analysis and synthesis of quantized feedback control systems, most results are developed for the case of a single quantizer (either measurement quantization or control signal quantization). In this technical note, we investigate the case of feedback control systems subject to both input and output quantization. This is motivated by the fact that it is common in remotely controlled systems that measurement and control signals are shared over a single digital network. More specifically, we consider a single-input single-output linear system with memoryless logarithmic quantizers. We firstly show that the output feedback quadratic stabilization problem in this setting can be addressed with no conservatism by means of a sector bound approach. Secondly, we provide a sufficient condition for quadratic stabilization via the solution of a scaled $\boldsymbol{H}_{\infty}$ control problem. Finally, we analyze a problem of bandwidth allocation in the communication channel for finite-level input and output quantizers.
\end{abstract}

Index Terms-Logarithmic quantizer, quadratic stabilization, quantized feedback systems, sector bound condition.

\section{INTRODUCTION}

The study of quantization errors in digital control systems has been an important area of research ever since digital controllers have been employed in feedback systems. Early works on quantized feedback

Manuscript received October 14, 2009; revised June 02, 2009. First published February 02, 2010; current version published March 10, 2010. This work was supported in part by "Conselho Nacional de Desenvolvimento Científico e Tecnológico, CNPq", Brazil, under grants 47.1739/2006-5, 30.2741/2005-4/PQ, 303.440/2008-2/PQ, and 20.0018/2008-6, and by ARC Centre for Complex Dynamic Systems and Control, Australia. Recommended by Associate Editor D. Liberzon.

D. F. Coutinho is with the Group of Automation and Control Systems, Faculty of Engineering, PUCRS, Porto Alegre RS 90619-900, Brazil (e-mail: dcoutinho@pucrs.br).

$\mathrm{M}$. Fu is with the School of Electrical Engineering and Computer Science, University of Newcastle, Callaghan, NSW 2308, Australia (e-mail: minyue. fu@newcastle.edu.au).

C. E. de Souza is with the Department of Systems and Control, Laboratório Nacional de Computação Científica, LNCC/MCT, Petrópolis, RJ 25651-075, Brazil (e-mail: csouza@lncc.br).

Color versions of one or more of the figures in this technical note are available online at http://ieeexplore.ieee.org.

Digital Object Identifier 10.1109/TAC.2010.2040497 control concentrated on analyzing the effects of quantization and ways to mitigate them [1]-[3]. The simplest approach to analyze the effects of quantized feedback control is to model the quantizer as sector bounded time-varying uncertainties and apply tools in the absolute stability theory.

Nowadays, many control systems are remotely implemented via communication channels with limited bandwidth which we will refer to as remote control systems. In such systems, the communication link is commonly shared by different applications and a natural issue is to minimize the quantity of information needed to be transmitted while achieving a certain closed-loop performance. In the last few years, many researchers have investigated this topic; see, e.g., [4]-[10]. From the results proposed in [7], a new line of research focuses on the quadratic stabilization problem of linear time-invariant (LTI) systems via quantized feedback using the so-called sector bound approach [9]. In this approach, the quantizer is assumed to be logarithmic and static (memoryless). One can cite several advantages in employing logarithmic quantizers such as the ease on addressing the quadratic stabilization problem, explicit coarsest quantization density formulae, and the nice feature of needing only a few bits to approximately achieve the performance of unquantized feedback systems.

In remote control systems, where the plant and the controller are physically distant, the sensor information (sent to the controller) and the control signal (fed back to the plant) are connected via a communication network. In other words, the feedback information is exchanged through a shared communication channel among the control system components (sensors, controller, actuator, etc.), and thus it is natural to suppose that both control and measurement signals are quantized [11], [12]. To date, very few works have addressed stability and stabilization problems for input and output quantized feedback systems as, for instance, [11]-[13]. On the other hand, in applications logarithmic quantizers are implemented with a finite number of quantization levels. Thus, we may be interested in minimizing the amount of information (i.e., the number of quantization levels) needed to assure (practical) stability when dealing with multiple (input and/or output) information resources sharing a common communication channel, which we refer to as the bandwidth allocation problem. In this setting, in [14] the resource (or bandwidth in our context) allocation problem for multiple input quantized control systems was addressed based on a modified $\mu$-synthesis problem.

In this technical note, we study the quantization effects on remote control systems as illustrate in Fig. 1. We assume that the system and the controller are physically separated and both the sensor and actuator share a communication channel. The sensor information is quantized (via quantizer 1) and sent to the controller, and similarly the control signal is quantized (via quantizer 2) and sent to the plant. In this setup, we follow a control theory perspective rather than information theory, in the sense that the quantizers are independent, static and require no dynamic encoding/decoding. This setting contrasts with information theory based approaches, where the two quantizers can be lumped into one, but this would mean using dynamic quantization and a "smart" processing before and after each quantizer (encoding and decoding). Notice that the proposed setup resembles a natural controller implementation framework. In this scenario, we extend the sector bound approach [9] to cope with input and output quantization for single-input, single-output (SISO) linear time-invariant systems under output feedback. We show that the problem of quadratic stability of the quantized system can be addressed with no conservatism by means of the sector bound approach. This result converts the quantized quadratic stabilization problem into a robust control problem, leading to a sufficient quadratic stabilization condition for the quantized system in terms 


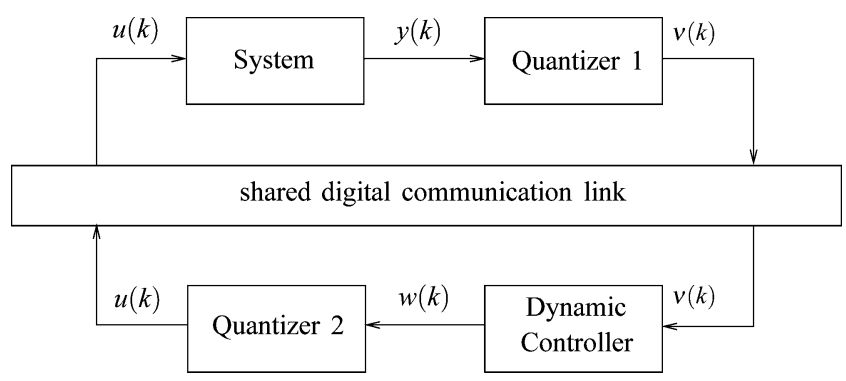

Fig. 1. Input and output quantized feedback control systems.

of a scaled $H_{\infty}$ control problem. Moreover, supposing a practical (finite-level) implementation of both quantizers, we analyze a bandwidth allocation problem aiming at minimizing the total number of bits transmitted over the communication channel.

This technical note is organized as follows. The quantized feedback problem to be addressed is stated in Section II; a key result on the sector bound approach for quantized output feedback systems with a single quantizer is reviewed in Section III; the main results of this technical note on input and output quantized feedback are developed in Section IV; concluding remarks are presented in Section V.

\section{Problem Statement}

Consider the quantized feedback system in Fig. 1. The system to be controlled is modeled by

$$
x(k+1)=A x(k)+B u(k), \quad y(k)=C x(k)
$$

where $A \in \mathbb{R}^{n \times n}, B \in \mathbb{R}^{n}, C \in \mathbb{R}^{1 \times n}, x$ is the state, $u$ is the control signal and $y$ is the measurement. The dynamic feedback controller is given by

$$
\begin{aligned}
\xi(k+1) & =A_{c} \xi(k)+B_{c} v(k), \\
w(k) & =C_{c} \xi(k)+D_{c} v(k) .
\end{aligned}
$$

The two quantizers are modeled by

$$
v(k)=Q_{1}(y(k)) \text { and } u(k)=Q_{2}(w(k))
$$

where $Q_{1}(\cdot)$ and $Q_{2}(\cdot)$ are static logarithmic quantizers with quantization densities $\rho_{1}$ and $\rho_{2}$, respectively.

Without loss of generality, we assume that $(A, B, C)$ and $\left(A_{c}, B_{c}, C_{c}, D_{c}\right)$ are minimal realizations and let $G(z)$ and $H(z)$ be their transfer functions, i.e.,

$$
\begin{aligned}
& G(z)=C(z I-A)^{-1} B \\
& H(z)=C_{c}\left(z I-A_{c}\right)^{-1} B_{c}+D_{c} .
\end{aligned}
$$

A logarithmic quantizer $Q(\cdot)$ with quantization density $\rho \in(0,1)$ has quantization levels given by

$$
\mathcal{V}=\left\{ \pm m_{j}: m_{j}=\rho^{j} \mu, j=0, \pm 1, \pm 2, \ldots\right\} \cup\{0\}
$$

where $\mu>0$ is a scaling parameter. A small $\rho$ implies coarse quantization and a large $\rho$ means dense quantization. The quantizer $Q(\cdot)$ is depicted in Fig. 2 and is defined as follows:

$$
Q(\varepsilon)= \begin{cases}\rho^{j} \mu, & \text { if } \frac{\rho^{j} \mu}{1+\delta} \leq \varepsilon<\frac{\rho^{j} \mu}{1-\delta}, j=0, \pm 1, \pm 2, \ldots \\ 0, & \text { if } \varepsilon=0 \\ -Q(-\varepsilon), & \text { if } \varepsilon<0\end{cases}
$$

with $\delta=(1-\rho) /(1+\rho)$.

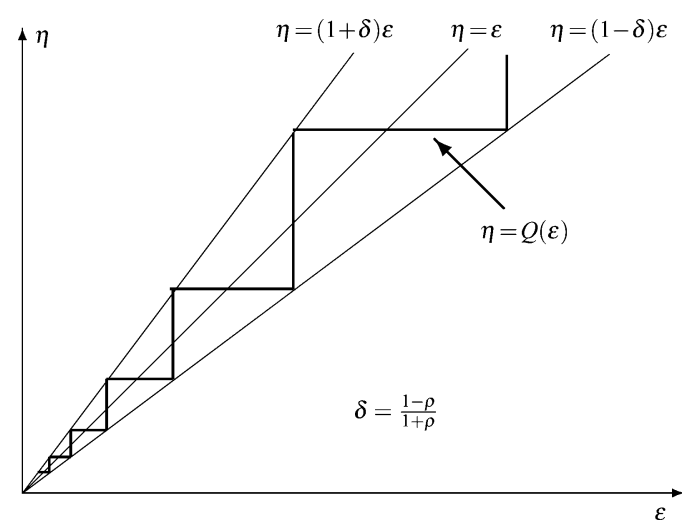

Fig. 2. Logarithmic quantizer (shown for positive input values only).

The closed-loop system can be represented by

$$
\left\{\begin{array}{l}
x(k+1)=A x(k)+B Q_{2}\left(C_{c} \xi(k)+D_{c} Q_{1}(C x(k))\right) \\
\xi(k+1)=A_{c} \xi(k)+B_{c} Q_{1}(C x(k))
\end{array}\right.
$$

which can be rewritten as

$$
\bar{x}(k+1)=f\left(x(k), \xi(k), \delta_{1}, \delta_{2}\right)
$$

where

$$
\begin{aligned}
\bar{x} & =\left[\begin{array}{l}
x^{T} \\
\xi^{T}
\end{array}\right]^{T}, \\
f\left(x, \xi, \delta_{1}, \delta_{2}\right) & =\left[\begin{array}{c}
A x+B Q_{2}\left(C_{c} \xi+D_{c} Q_{1}(C x)\right) \\
A_{c} \xi+B_{c} Q_{1}(C x)
\end{array}\right] .
\end{aligned}
$$

In this technical note, we assume that the input and output quantizers are independent with possibly different quantization densities. Under these conditions, we address the quadratic stability problem of the quantized closed-loop feedback system in (8). Furthermore, assuming finite-level quantizers, we study a bandwidth allocation problem in the sense that the quantization densities $\rho_{i}$ of the quantizers $Q_{i}(\cdot), i=1,2$, are chosen to minimize the communication channel bandwidth.

\section{PReVIOUS RESUlts}

In this section, we review a key result in [9] where the quadratic stabilization problem of SISO linear feedback systems with a single quantizer is solved via the sector bound approach and $H_{\infty}$ control.

Notice from Fig. 2 that a quantizer $Q(\varepsilon)$ can be bounded by a sector $(1+\Delta) \varepsilon$, where $\Delta \in[-\delta, \delta]$ and consider two possible configurations involving the system (1), the controller (2) and a quantizer:

Configuration I: The measurement is quantized, i.e., $v(k)=$ $Q_{1}(y(k))$, but the control signal is not, i.e., $u(k)=w(k)$; and

Configuration II: The control signal is quantized, i.e., $u(k)=$ $Q_{2}(w(k))$, but the measurement is not, i.e., $v(k)=y(k)$.

Considering the controller in (2) for either Configuration I or II, we extend a result in [9] as follows.

Theorem 1: Consider system (1) and a single quantizer in Configuration I or II. For a given quantizer density $\rho$, this system is quadratically stabilizable via controller (2) if and only if the auxiliary system

$$
\left\{\begin{array}{l}
x(k+1)=A x(k)+B w(k) \\
v(k)=(1+\Delta) C x(k),|\Delta| \leq \delta
\end{array}\right.
$$

for Configuration I, or

$$
\left\{\begin{array}{l}
x(k+1)=A x(k)+B(1+\Delta) w(k) \\
v(k)=C x(k),|\Delta| \leq \delta
\end{array}\right.
$$




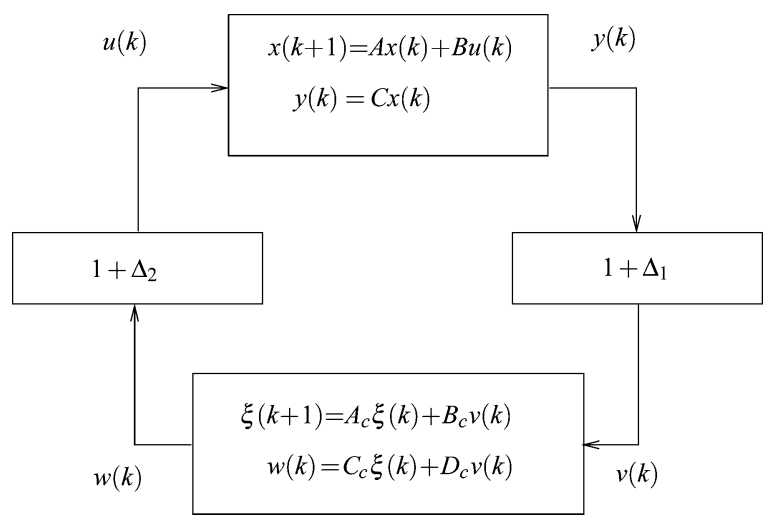

Fig. 3. Auxiliary system.

for Configuration II, is quadratically stabilizable via controller (2), where $\delta$ and $\rho$ are related as in (6). Moreover, for both configurations, the supremum $\delta_{\text {sup }}$ of the sector bound $\delta$ for quadratic stabilization, which provides the coarsest quantization density $\rho_{\text {inf }}$, is given by

$$
\delta_{\mathrm{sup}}=\left(\inf _{\mathcal{C}}\|\bar{G}(z)\|_{\infty}\right)^{-1}
$$

where

$$
\bar{G}(z)=\frac{G(z) H(z)}{1-G(z) H(z)}, \quad \mathcal{C}=\left(A_{c}, B_{c}, C_{c}, D_{c}\right)
$$

Proof: The proof of the equivalence between the quadratic stability of the quantized and the auxiliary closed-loop systems can be found in [9, Theorem 3.2] The result on $\delta_{\text {sup }}$ follows by noting that since $G(z) H(z)=H(z) G(z)$, then for Configurations I and II the closed-loop system can be written as an open-loop transfer function $G(z) H(z)$ and a feedback loop $(1+\Delta)$. Finally, the solution to $\delta_{\text {sup }}$ follows from the equivalence between quadratic stability and $H_{\infty}$ control, see, e.g., [15].

Remark 1: Note that in [9], the authors addressed the Configuration II problem by means of a deadbeat observer yielding a result in [9, Theorem 3.1] different from the result stated in Theorem 1 as above, which considers a dynamic LTI controller for both Configurations I and II.

\section{MAIN RESUlTS}

In this section, we extend the result of Theorem 1 to the double quantizer stabilization problem as defined in the closed-loop system in Fig. 1. Firstly, we show that the quadratic stability analysis problem can be tackled with no conservatism by two sector bound conditions. Secondly, a sufficient condition for output feedback quadratic stabilization is derived in terms of a standard $H_{\infty}$ control problem. Finally, we consider a bandwidth allocation problem as an application of the quadratic stabilization result.

\section{A. Input and Output Sector Bound Conditions}

Consider a Lyapunov function candidate $V(\bar{x})=\bar{x}^{T} P \bar{x}$, with $P=$ $P^{T}>0$, for the closed-loop system (7), or (8), and define

$\Phi\left(x, \xi, \delta_{1}, \delta_{2}, \varepsilon\right)$

$$
=f\left(x, \xi, \delta_{1}, \delta_{2}\right)^{T} \operatorname{Pf}\left(x, \xi, \delta_{1}, \delta_{2}\right)-(1-\varepsilon) \bar{x}^{T} P \bar{x}
$$

where $\varepsilon$ is a positive scalar. Then, along the trajectory of (7) we have

$$
V(\bar{x}(k+1))-V(\bar{x}(k))<\Phi\left(x(k), \xi(k), \delta_{1}, \delta_{2}, \varepsilon\right) .
$$

Hence, (7) is quadratically stable if and only if there exists some $P=P^{T}>0$ and $\varepsilon>0$ such that

$$
\Phi\left(x, \xi, \delta_{1}, \delta_{2}, \varepsilon\right) \leq 0, \forall x, \xi .
$$

Define

$$
\begin{aligned}
\bar{A}\left(\Delta_{1}, \Delta_{2}\right)= & {\left[\begin{array}{cc}
A & 0 \\
0 & A_{c}
\end{array}\right] } \\
& \left.\left.+\left[\begin{array}{c}
B\left(1+\Delta_{2}\right)\left(\left[0 C_{c}\right]+D_{c}\left(1+\Delta_{1}\right)[C\right. \\
C
\end{array}\right]\right)\right]
\end{aligned}
$$

and

$$
\Omega\left(\Delta_{1}, \Delta_{2}\right)=\bar{A}\left(\Delta_{1}, \Delta_{2}\right)^{T} P \bar{A}\left(\Delta_{1}, \Delta_{2}\right)-P .
$$

The first result of this section is given below.

Theorem 2: Consider the closed-loop quantized system (7) and some given $P=P^{T}>0$. Then, (16) holds for some $\varepsilon>0$ (i.e., (7) is quadratically stable) if and only if

$$
\Omega\left(\Delta_{1}, \Delta_{2}\right)<0, \quad \forall\left|\Delta_{1}\right| \leq \delta_{1},\left|\Delta_{2}\right| \leq \delta_{2} .
$$

The proof is given in the Appendix.

Remark 2: Theorem 2 implies that the quadratic stabilization problem for the input-output quantized feedback system can be transformed, with no conservatism, into a standard robust control problem. This result is strong in the sense that the quadratic stability of the uncertain system $\bar{x}(k+1)=\bar{A}\left(\Delta_{1}, \Delta_{2}\right) \bar{x}(k)$, with $\left|\Delta_{i}\right| \leq \delta_{i}, i=1,2$, is a necessary and sufficient condition for the quadratic stability of the quantized closed-loop system of (1) and (2).

\section{B. Quadratic Stabilization}

From Theorem 2, it follows that the quadratic stability of the closedloop system of (1)-(3) is equivalent to the quadratic stability of the following auxiliary system:

$$
\left\{\begin{array}{l}
x(k+1)=A x(k)+B\left(1+\Delta_{2}\right) w(k) \\
y(k)=C x(k) \\
\xi(k+1)=A_{c} \xi(k)+B_{c}\left(1+\Delta_{1}\right) y(k) \\
w(k)=C_{c} \xi(k)+D_{c}\left(1+\Delta_{1}\right) y(k)
\end{array}\right.
$$

as illustrated in Fig. 3, where $\left|\Delta_{1}\right| \leq \delta_{1},\left|\Delta_{2}\right| \leq \delta_{2}$, and the parameters $\delta_{i}$ are related to the quantization densities $\rho_{i}$ via

$$
\delta_{i}=\frac{\left(1-\rho_{i}\right)}{\left(1+\rho_{i}\right)}, i=1,2 .
$$

Now, define the following auxiliary notation:

$$
\begin{aligned}
\hat{A} & =\left[\begin{array}{cc}
A+B D_{c} C & B C_{c} \\
B_{c} C & A_{c}
\end{array}\right], \hat{B}=\left[\begin{array}{cc}
B D_{c} & B \\
B_{c} & 0
\end{array}\right], \\
\hat{C} & =\left[\begin{array}{cc}
C & 0 \\
D_{c} C & C_{c}
\end{array}\right], \hat{D}=\left[\begin{array}{cc}
0 & 0 \\
D_{c} & 0
\end{array}\right], \\
q(k) & =\left[\begin{array}{l}
q_{1} \\
q_{2}
\end{array}\right], p(k)=\left[\begin{array}{l}
p_{1} \\
p_{2}
\end{array}\right], q_{1}=y(k), \\
q_{2} & =w(k), p_{1}=\Delta_{1} q_{1}, p_{2}=\Delta_{2} q_{2} .
\end{aligned}
$$

Through standard linear fractional transformations [16], system (20) can be recast in the form

$$
\left\{\begin{array}{l}
\bar{x}(k+1)=\hat{A} \bar{x}(k)+\hat{B} p(k) \\
q(k)=\hat{C} \bar{x}(k)+\hat{D} p(k) \\
p(k)=\hat{\Delta} q(k), \hat{\Delta}=\operatorname{diag}\left\{\Delta_{1}, \Delta_{2}\right\}
\end{array}\right.
$$

where $\left|\Delta_{i}\right| \leq \delta_{i}, i=1,2$. 
Let $\hat{G}(z)$ be the transfer function matrix from $p$ to $q$ of the open-loop system in (24), i.e.,

$$
\hat{G}(z)=\hat{C}(z I-\hat{A})^{-1} \hat{B}+\hat{D} .
$$

From well known quadratic stability results [15], system (24) is quadratically stable if

$$
\left\|\mathcal{T} \hat{G}(z) \mathcal{T}^{-1} \operatorname{diag}\left\{\delta_{1}, \delta_{2}\right\}\right\|_{\infty}<1
$$

where $\mathcal{T}$ is any invertible diagonal matrix. Without loss of generality, we can take $\mathcal{T}=\operatorname{diag}\{1, \tau\}, \tau>0$.

The discussion above is summarized in the following theorem.

Theorem 3: Consider system (1) and quantizers as in (3) with given densities $\rho_{1}$ and $\rho_{2}$. This system is quadratically stabilizable via controller (2) if and only if the auxiliary system

$$
\left\{\begin{aligned}
x(k+1) & =A x(k)+B\left(1+\Delta_{2}\right) w(k),\left|\Delta_{2}\right| \leq \delta_{2} \\
v(k) & =\left(1+\Delta_{1}\right) C x(k),\left|\Delta_{1}\right| \leq \delta_{1}
\end{aligned}\right.
$$

is quadratically stabilizable via controller (2). Moreover, a sufficient condition for the latter is that (26) holds, where $\hat{G}(z)$ is as in (25), $\mathcal{T}=\operatorname{diag}\{1, \tau\}, \tau>0$, and $\delta_{1}, \delta_{2}$ as in (21).

Remark 3: For a single quantizer, Theorem 3 becomes equivalent to Theorem 1, since the small-gain theorem is necessary and sufficient for quadratic stability with a single uncertainty block [16].

Remark 4: It follows from Theorem 3 that a quadratically stabilizing output feedback controller $\mathcal{C}$ for system (1) can be designed via the scaled $H_{\infty}$ control problem in (26). Note that both the controller and the scaling $\tau>0$ need to be jointly designed, leading to a non-convex problem. Nevertheless, we can perform a line search on $\tau$ and apply standard LMI approach of $H_{\infty}$ control to determine the controller, as for instance, the approach in [17].

Remark 5: Theorem 3 does not address the issue of obtaining upper bounds for $\delta_{1}$ and $\delta_{2}$. Nevertheless, these bounds can be determined numerically via a gridding technique. To this end, we need to define a procedure to perform the gridding. A possible way is as follows. We consider only one quantizer, by either setting $\delta_{1}=0$ or $\delta_{2}=0$, then determine upper bounds $\bar{\delta}_{1}$ or $\bar{\delta}_{2}$ on $\delta_{1}$ or $\delta_{2}$, respectively, by means of Theorem 1. This implies that for any $\delta_{i}<\bar{\delta}_{i}, i=1$ or $i=2$, we can find an upper bound for $\delta_{j}, j \neq i$ such that (26) is satisfied. The latter procedure leads to a bounding curve in the $\left(\delta_{1}, \delta_{2}\right)$ space.

\section{Bandwidth Allocation}

To demonstrate the applicability of the results in the previous subsections, we consider a bandwidth allocation problem where the communication link in Fig. 1 has a fixed bandwidth which is to be allocated between the two quantizers. To motivate the bandwidth allocation problem, we assume that the logarithmic quantizers $Q_{1}$ and $Q_{2}$ are truncated so that their input ranges are $\left[\sigma_{i}, 1 / \sigma_{i}\right]$ for sufficiently small $\sigma_{i}>0, i=1,2$, respectively. This leads to the notion of practical stability as studied in [7], which implies that the system state trajectory will asymptotically converge to a negligibly small neighborhood of the origin, provided that the initial state is not very large such that the quantizers will never saturate (see [7] for further details). From this assumption, it follows that (26) is still approximately valid. The requirement that $\sigma_{1}$ and $\sigma_{2}$ are sufficiently small is reasonable because: (i) this is a common practice for digital control implementation with linear (or uniform) quantizers; and (ii) logarithmic quantizers have far larger dynamic ranges than linear quantizers.
With the assumption that the logarithmic quantizer $Q_{i}$ has an input range $\left[\sigma_{i}, 1 / \sigma_{i}\right]$, the number of quantization levels in this range is approximately given by

$$
N_{i} \approx \frac{2 \ln \left(\frac{1}{\sigma_{i}}\right)}{\ln \left(\frac{1}{\rho_{i}}\right)}
$$

following from the simple fact that [7]:

$$
\lim _{\sigma_{i} \rightarrow 0} \frac{N_{i}}{\ln \left(\frac{1}{\sigma_{i}}\right)}=\frac{2}{\ln \left(\frac{1}{\rho_{i}}\right)} .
$$

Thus, the total number of bits $N_{b}$ to be transmitted is given by

$$
\begin{aligned}
N_{b} & =\log \left(2 N_{1}\right)+\log \left(2 N_{2}\right) \\
& \approx \log \left(\frac{4 \ln \left(\frac{1}{\sigma_{1}}\right)}{\ln \left(\frac{1}{\rho_{1}}\right)} \cdot \frac{4 \ln \left(\frac{1}{\sigma_{2}}\right)}{\ln \left(\frac{1}{\rho_{2}}\right)}\right) .
\end{aligned}
$$

Our bandwidth allocation problem aims to minimize the right-hand side of (28), where $\sigma_{1}$ and $\sigma_{2}$ are chosen a priori to be sufficiently small so that (26) is approximately valid. Note that this minimization should be carried out subject to (26). Thus, we consider in the sequel the following cost function:

$$
\begin{aligned}
J\left(\delta_{1}, \delta_{2}\right) & =\frac{1}{\ln \left(\frac{1}{\rho_{1}}\right) \ln \left(\frac{1}{\rho_{2}}\right)} \\
& =\frac{1}{\ln \left(\frac{1+\delta_{1}}{1-\delta_{1}}\right) \ln \left(\frac{1+\delta_{2}}{1-\delta_{2}}\right)} .
\end{aligned}
$$

Hence, the problem becomes to determine $\delta_{1}$ and $\delta_{2}$ by means of the following optimization problem:

$$
\min _{\mathcal{C}, \mathcal{T}, \delta_{1}, \delta_{2}} J\left(\delta_{1}, \delta_{2}\right) \text { subject to }(26) .
$$

The pair $\left(\delta_{1}, \delta_{2}\right)$ that minimizes (30) can be obtained by gridding $\delta_{1}$ from 0 up to some upper bound $\delta_{\text {sup }}$, which is the maximum admissible $\delta$ for one quantizer, and numerically compute $\delta_{2}, \mathcal{C}$ and $\mathcal{T}=$ $\operatorname{diag}\{1, \tau\}, \tau>0$, such that (26) holds. The values $\delta_{1}^{*}$ and $\delta_{2}^{*}$ such that $J\left(\delta_{1}, \delta_{2}\right)$ is minimum give the best quantization densities $\rho_{i}^{*}=$ $\left(1-\delta_{i}^{*}\right)\left(1+\delta_{i}^{*}\right)^{-1}, i=1,2$.

Remark 6: Note that the total number of bits $N_{b}$ as given in (28) also depends on the quantizers' parameters $\sigma_{1}$ and $\sigma_{2}$ and their optimal values should be determined while ensuring practical stability.

To illustrate the above result, consider the following system borrowed from [9, Example 3.1]:

$$
G(z)=\frac{z-3}{z(z-2)}
$$

Considering the above system with one quantizer, by Theorem 1 the supremum $\delta$ for output feedback quadratic stabilization is $\delta_{\text {sup }}=0.1$. To determine the optimal $\delta_{1}^{*}$ and $\delta_{2}^{*}$, we grid $\delta_{1}$ from 0 to $\delta_{\text {sup }}$ and for each $\delta_{1}$ determine $\delta_{2} \in\left(0, \delta_{\text {sup }}\right)$ along with $\left(A_{c}, B_{c}, C_{c}, D_{c}\right)$ and $\tau>0$ such that $J\left(\delta_{1}, \delta_{2}\right)$ is minimized subject to

$$
\left\|\operatorname{diag}\{1, \tau\} \hat{G}(z) \operatorname{diag}\left\{1, \frac{1}{\tau}\right\} \operatorname{diag}\left\{\delta_{1}, \delta_{2}\right\}\right\|_{\infty}<1 .
$$

The results obtained for $J\left(\delta_{1}, \delta_{2}\right)$ are displayed in Fig. 4. It follows from these results that the minimum $J\left(\delta_{1}, \delta_{2}\right)$ is achieved with $\delta_{1}^{*} \cong \delta_{2}^{*} \cong 0.05$ (the point that minimizes the curve in Fig. 4), which corresponds to $\rho_{1}^{*}=\rho_{2}^{*} \cong 0.9$. 


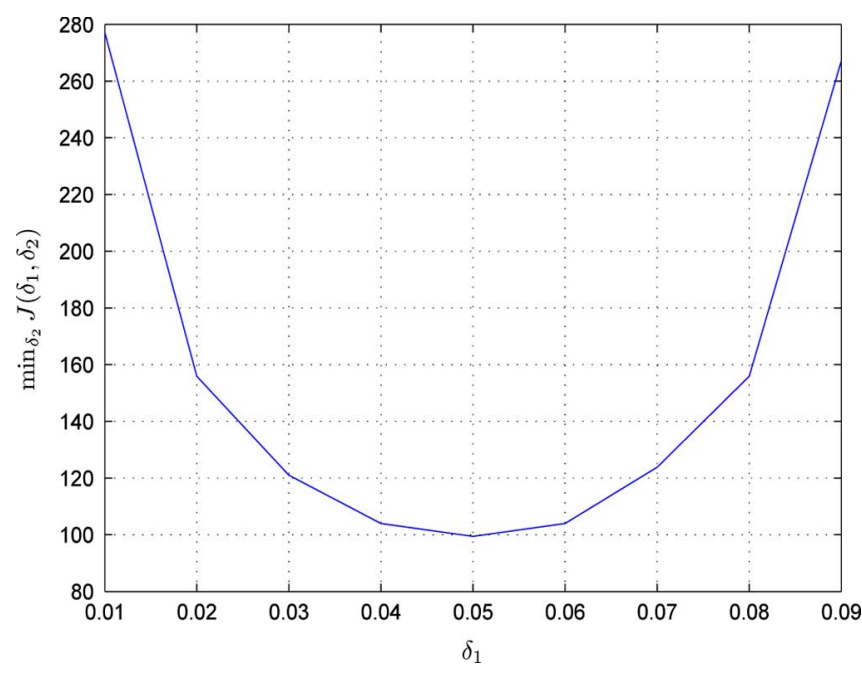

Fig. 4. Cost function $\boldsymbol{J}\left(\boldsymbol{\delta}_{1}, \boldsymbol{\delta}_{2}\right)$.

The result derived from Fig. 4 illustrates how the optimization problem in (30) can be applied to determine the quantization densities. As pointed out in Remark $6, N_{b}$ is also dependent on the quantizers' parameters $\sigma_{1}$ and $\sigma_{2}$, and to simplify the problem we have assumed that $\sigma_{1}$ and $\sigma_{2}$ are sufficiently small to guarantee practical stability. Note that the largest values of $\sigma_{1}$ and $\sigma_{2}$ guaranteeing practical stability depend on the set of admissible initial states and the estimation of such values is beyond the scope of this technical note. Nevertheless, considering the controller designed as above for the optimal $\delta_{1}^{*}=\delta_{2}^{*}=0.05$, we have determined through numerical simulations the largest $\sigma_{1}$ and $\sigma_{2}$ guaranteeing practical stability for any initial state inside the ellipsoidal region

$$
X_{0}=\left\{x \in \mathbb{R}^{2}: 4 x_{1}^{2}-5.1 x_{1} x_{2}+2.1 x_{2}^{2} \leq 1\right\} \text {. }
$$

The results we obtained are $\sigma_{1} \leq 1 / 12$ and $\sigma_{2} \leq 1 / 8$, which lead to $N_{1} \geq 54$ and $N_{2} \geq 46$. For this example, we need more information regarding the measurement than the control signal to ensure practical stability, but the numbers of bits for both quantizers are the same (i.e., $N_{b_{1}}=N_{b_{2}} \geq 7$ ).

\section{CONCLUSION}

This technical note has extended the sector bound approach to cope with input and output quantized feedback linear systems. The contribution of this technical note is as follows. Firstly, we have shown that the problem of quadratic stabilization via quantized output feedback can be addressed with no conservatism through an auxiliary uncertain system with two sector bound conditions. Secondly, we have derived a sufficient quadratic stabilization condition in terms of a scaled $H_{\infty}$ control problem. Thirdly, we have tackled a bandwidth allocation problem in the sense of minimizing the total number of bits transmitted over the communication channel.

\section{APPENDIX}

First, we introduce three lemmas needed in the proof of Theorem 2. In the sequel, we assume that $P=P^{T}>0$ and, without loss of generality, we set $\mu=1$ for the quantizers $Q_{1}(\cdot)$ and $Q_{2}(\cdot)$.

Lemma 1: Suppose (16) holds. Then

$$
\Phi\left(x, \xi, \delta_{1}-\varepsilon_{1}, \delta_{2}, \varepsilon\right) \leq 0, \forall x, \xi
$$

when $\varepsilon_{1}>0$ is sufficiently small.

Proof: We first consider any $x$ such that $y=C x$ is fixed and $y \in\left[1 /\left(1+\delta_{1}\right), 1 /\left(1-\delta_{1}\right)\right)$. In this case, $Q_{1}(C x)=1$. Next, let $g\left(y, \delta_{2}, \varepsilon\right)=\max _{x, \xi, C x=y} \Phi\left(x, \xi, \delta_{1}, \delta_{2}, \varepsilon\right)$. Note that $g\left(y, \delta_{2}, \varepsilon\right)$ does not depend on $\delta_{1}$. From (7), it is clear that $g\left(y, \delta_{2}, \varepsilon\right) \leq 0$ for all $y \in\left[1 /\left(1+\delta_{1}\right), 1 /\left(1-\delta_{1}\right)\right)$.

Now consider the case that $\delta_{1}$ is reduced to $\tilde{\delta}_{1}=\delta_{1}-\varepsilon_{1}$ with $0<$ $\varepsilon_{1}<\delta_{1}$. For $y \in\left[1 /\left(1+\tilde{\delta}_{1}\right), 1 /\left(1-\tilde{\delta}_{1}\right)\right)$, we still have $Q_{1}(y)=1$ and $g\left(y, \delta_{2}, \varepsilon\right)$ remains the same and hence $g\left(y, \delta_{2}, \varepsilon\right) \leq 0$. That is,

$$
\Phi\left(x, \xi, \tilde{\delta}_{1}, \delta_{2}, \varepsilon\right) \leq 0, \forall x, \xi: C x \in\left[\frac{1}{\left(1+\tilde{\delta}_{1}\right)}, \frac{1}{\left(1-\tilde{\delta}_{1}\right)}\right] .
$$

Let $\tilde{\rho}_{1}=\left(1-\tilde{\delta}_{1}\right) /\left(1+\tilde{\delta}_{1}\right)$. For $y=C x \in\left[\tilde{\rho}_{1}^{i} /\left(1+\tilde{\delta}_{1}\right), \tilde{\rho}_{1}^{i} /(1-\right.$ $\left.\tilde{\delta}_{1}\right)$ ], we have $Q_{1}(C x)=\tilde{\rho}_{1}^{i}$ and

$$
\Phi\left(x, \xi, \tilde{\delta}_{1}, \delta_{2}, \varepsilon\right)=\tilde{\rho}_{1}^{i} \Phi\left(\hat{x}, \hat{\xi}, \tilde{\delta}_{1}, \delta_{2}, \varepsilon\right)
$$

where $\hat{x}=x \tilde{\rho}_{1}^{-i}$ and $\hat{\xi}=\xi \tilde{\rho}_{1}^{-i}$ with $C \hat{x} \in\left[1 /\left(1+\tilde{\delta}_{1}\right), 1 /\left(1-\tilde{\delta}_{1}\right)\right)$. Using (32) (with $\hat{x}$ and $\hat{\xi}$ in lieu of $x$ and $\xi$ ), we get $\Phi\left(x, \xi, \tilde{\delta}_{1}, \delta_{2}, \varepsilon\right) \leq$ $0, \forall x, \xi: C x \in\left[\tilde{\rho}_{1}^{i} /\left(1+\tilde{\delta}_{1}\right), \tilde{\rho}_{1}^{i} /\left(1-\tilde{\delta}_{1}\right)\right]$ for all $i$. Using the facts that $(0, \infty)$ is covered by the union of all $\left[\tilde{\rho}_{1}^{i} /\left(1+\tilde{\delta}_{1}\right), \tilde{\rho}_{1}^{i} /\left(1-\tilde{\delta}_{1}\right)\right]$ and that $\Phi\left(x, \xi, \delta_{1}, \delta_{2}, \varepsilon\right)$ is an even function of $x$, the claim in the lemma follows.

Lemma 2: Given a logarithmic quantizer $Q(\cdot)$ in (6) with quantization density $\rho$, let $\delta$ be given as in (6) and define

$$
\Delta(v)=\frac{Q(v)}{v-1}, \quad v \neq 0
$$

Then, the following properties hold:

1) $|\Delta(v)| \leq \delta$ for any $v \neq 0$.

2) For any $\Delta_{0} \in[-\delta, \delta)$, there exists a unique solution $v_{0}>0$ to $\Delta(v)=\Delta_{0}$ in $v \in[1 /(1+\delta), 1 /(1-\delta))$. Moreover, all the solutions of $v$ in $(0, \infty)$ are given by $\pm \rho^{i} v_{0}, i=0, \pm 1, \pm 2, \ldots$

These properties are easily verified, so the proof is omitted.

Lemma 3 ([18]): Given any irrational number $\alpha$, there exists a sequence $\left(n_{k}, d_{k}\right), k=1,2, \ldots$, such that $n_{k}$ and $d_{k}$ are coprime, $d_{k} \rightarrow \infty$ as $k \rightarrow \infty$ and

$$
\left|\frac{n_{k}}{d_{k}}-\alpha\right| \leq \frac{1}{d_{k}}, \quad \forall k=1,2, \ldots
$$

Proof of Theorem 2: We first show the sufficiency. Suppose $\Omega\left(\Delta_{1}, \Delta_{2}\right)<0$ for all $\left|\Delta_{1}\right| \leq \delta_{1}$ and $\left|\Delta_{2}\right| \leq \delta_{2}$. By continuity, there exists some small $\varepsilon>0$ such that $\Omega\left(\Delta_{1}, \Delta_{2}\right)+\varepsilon P \leq 0$ for all $\left|\Delta_{1}\right| \leq \delta_{1}$ and $\left|\Delta_{2}\right| \leq \delta_{2}$. Now, a direct consequence of Lemma 2 is that $Q_{1}(v)=\left(1+\Delta_{1}(v)\right) v$ with $\left|\Delta_{1}(v)\right| \leq \delta_{1}$ for any $v$. A similar result holds for $Q_{2}(\cdot)$. Hence, we can write

$$
\Phi\left(x, \xi, \delta_{1}, \delta_{2}, \varepsilon\right)=\left[\begin{array}{l}
x \\
\xi
\end{array}\right]^{T}\left(\Omega\left(\Delta_{1}\left(v_{1}\right), \Delta_{2}\left(v_{2}\right)\right)+\varepsilon P\right)\left[\begin{array}{l}
x \\
\xi
\end{array}\right]
$$

with $\left|\Delta_{1}\left(v_{1}\right)\right| \leq \delta_{1}$ and $\mid \Delta_{2}\left(v_{2}\right) \leq \delta_{2}$, where $v_{1}=C x$ and $v_{2}=$ $C_{c} \xi+D_{c}\left(1+\Delta_{1}\left(v_{1}\right)\right) v_{1}$. Hence, for all $x, \xi$ and for the chosen $\varepsilon>0$, we obtain $\Phi\left(x, \xi, \delta_{1}, \delta_{2}, \varepsilon\right) \leq 0$.

To prove the necessity, we assume that $\Phi\left(x, \xi, \delta_{1}, \delta_{2}, \varepsilon\right) \leq 0$ for all $x$ and $\xi$, for some $\varepsilon>0$. The proof is done by contradiction. To this end, we assume that there exist some $\left|\Delta_{1}^{0}\right| \leq \delta_{1},\left|\Delta_{2}^{0}\right| \leq \delta_{2}$ and nonzero $\bar{x}_{0}=\left[x_{0}^{T}, \xi_{0}^{T}\right]^{T}$ such that $\bar{x}_{0}^{T} \Omega\left(\Delta_{1}^{0}, \Delta_{2}^{0}\right) \bar{x}_{0} \geq \overline{0}$. By continuity, this implies that there exist some $\left|\Delta_{1}^{0}\right|<\delta_{1}$ and $\left|\Delta_{2}^{0}\right|<\delta_{2}$ (obtained by "shrinking" the previous $\Delta_{1}^{0}$ and $\Delta_{2}^{0}$ a bit if necessary) such that

$$
\bar{x}_{0}^{T}\left(\Omega\left(\Delta_{1}^{0}, \Delta_{2}^{0}\right)+\left(\frac{\varepsilon}{3}\right) P\right) \bar{x}_{0} \geq 0 .
$$


Also by continuity, in the event that $C x_{0}=0$, we may perturb $x_{0}$ slightly so that $C x_{0}$ become nonzero and (35) is relaxed to

$$
\bar{x}_{0}^{T}\left(\Omega\left(\Delta_{1}^{0}, \Delta_{2}^{0}\right)+\left(\frac{\varepsilon}{2}\right) P\right) \bar{x}_{0} \geq 0 .
$$

We need to show that (36) leads to a contradiction. We first consider the case where $\ln \rho_{2} / \ln \rho_{1}$ is an irrational number. Using Lemma 2 , we know that all the solutions to $\Delta_{1}\left(v_{1}\right)=\Delta_{1}^{0}$ are given by $\pm v_{1}^{0} \rho_{1}^{i}$, $i=0, \pm 1, \pm 2, \ldots$ for some $v_{1}^{0}>0$. Similarly, all the solutions to $\Delta_{2}\left(v_{2}\right)=\Delta_{2}^{0}$ are given by $\pm v_{2}^{0} \rho_{2}^{j}, j=0, \pm 1, \pm 2, \ldots$ for some $v_{2}^{0}>$ 0 .

Define $x^{(0)}=g_{0} x_{0}$ and $\xi^{(0)}=g_{0} \xi_{0}$, with $g_{0}=v_{1}^{0} / C x_{0}$. Then, we have $Q_{1}\left(C x^{(0)}\right)=Q_{1}\left(v_{1}^{0}\right)=\left(1+\Delta_{1}^{0}\right) C x^{(0)}$.

Denote $w^{0}=C_{c} \xi^{(0)}+D_{c}\left(1+\Delta_{1}^{0}\right) C x^{(0)}, \alpha=\ln \rho_{2} / \ln \rho_{1}$ and $\beta=\ln \left(v_{2}^{0} / w^{0}\right) / \ln \rho_{1}$.

By Lemma 3 , there exists a sequence of $\left(n_{k}, d_{k}\right)$ with the properties described in Lemma 3. We can always choose $m_{k}$ be such that $\left|m_{k} / d_{k}-\beta\right| \leq 1 / d_{k}$. Since $n_{k}$ and $d_{k}$ are coprime, there exists a unique solution of $\left(i_{k}, j_{k}\right)$ to

$$
i_{k} d_{k}-j_{k} n_{k}=m_{k}, \quad 0 \leq j_{k}<d_{k} .
$$

Using the above, we get

$$
\begin{aligned}
\left|i_{k}-j_{k} \alpha-\beta\right| & =\left|\left(i_{k}-j_{k} \frac{n_{k}}{d_{k}}-\frac{m_{k}}{d_{k}}\right)+j_{k}\left(\frac{n_{k}}{d_{k}}-\alpha\right)+\left(\frac{m_{k}}{d_{k}}-\beta\right)\right| \\
& \leq d_{k} \frac{1}{d_{k}^{2}}+\frac{1}{d_{k}}=\frac{2}{d_{k}} .
\end{aligned}
$$

It follows that $\left|i_{k} \ln \rho_{1}-j_{k} \ln \rho_{2}-\ln \left(v_{2}^{0} / w^{0}\right)\right| \leq \eta_{k}$, or, alternatively

$$
w^{0} \rho_{1}^{i_{k}}=e^{\eta_{k}} v_{2}^{0} \rho_{2}^{j_{k}}
$$

where $\left|\eta_{k}\right| \leq 2 \ln \rho_{1} / d_{k} \rightarrow 0$ as $k \rightarrow \infty$.

Now considering $x^{(k)}=\rho_{1}^{i_{k}} x^{(0)}, \xi^{(k)}=\rho_{1}^{i_{k}} \xi^{(0)}$ and using the definition of $w^{0}$, we get $Q_{2}\left(C_{c} \xi^{(k)}+D_{c} Q_{1}\left(C x^{(k)}\right)\right)=Q_{2}\left(w_{0} \rho_{1}^{i_{k}}\right)=$ $Q_{2}\left(e^{\eta_{k}} v_{2}^{0} \rho_{2}^{j_{k}}\right)=\rho_{2}^{j_{k}} Q_{2}\left(e^{\eta_{k}} v_{2}^{0}\right)$.

Since $\left|\Delta_{2}^{0}\right|<\delta_{2}$ (a strict inequality), we must have $v_{2}^{0} \in(1 /(1+$ $\left.\delta_{2}\right), 1 /\left(1-\delta_{2}\right)$ ) (an open interval). Hence, for sufficiently large $k, \eta_{k}$ will be sufficiently small, so $e^{\eta_{k}} v_{2}^{0} \in\left(1 /\left(1+\delta_{2}\right), 1 /\left(1-\delta_{2}\right)\right)$ and $Q_{2}\left(e^{\eta_{k}} v_{2}^{0}\right)=\left(1+\Delta_{2}\right) v_{2}^{0}$. Therefore, $Q_{2}\left(C_{c} \xi^{(k)}+D_{c} Q_{1}\left(C x^{(k)}\right)\right)=$ $\rho_{2}^{j_{k}}\left(1+\Delta_{2}^{0}\right) v_{2}^{0}=\left(1+\Delta_{2}^{0}\right) e^{-\eta_{k}} \rho_{1}^{i_{k}} w^{0}=e^{-\eta_{k}}\left(1+\Delta_{2}^{0}\right)\left(\left[0 C_{c}\right]+\right.$ $\left.D_{c}\left(1+\Delta_{1}^{0}\right)\left[\begin{array}{ll}C & 0\end{array}\right]\right) \bar{x}^{(k)}$.

Hence, we can write

$$
\Phi\left(x^{(k)}, \xi^{(k)}, \delta_{1}, \delta_{2}, \varepsilon\right)=\left(\bar{x}^{(k)}\right)^{T}\left(\Omega\left(\Delta_{1}^{0}, \Delta_{2}^{0}, \eta_{k}\right)+\varepsilon P\right) \bar{x}^{(k)}
$$

where $\Omega\left(\Delta_{1}^{0}, \Delta_{2}^{0}, \eta_{k}\right) \rightarrow \Omega\left(\Delta_{1}^{0}, \Delta_{2}^{0}\right), \quad k \rightarrow \infty$. Using $\bar{x}^{(k)}=$ $\rho_{1}^{i_{k}} g_{0} \bar{x}_{0}$ and (33), it follows from the above that:

$$
\Phi\left(x^{(k)}, \xi^{(k)}, \delta_{1}, \delta_{2}, \varepsilon\right) \geq\left(\frac{\varepsilon}{4}\right)\left(\bar{x}^{(k)}\right)^{T} P x^{(k)}>0
$$

for some sufficiently large $k$. This contradicts the assumption that $\Phi\left(x, \xi, \delta_{1}, \delta_{2}, \varepsilon\right) \leq 0$ for all $x, \xi$. This contradiction implies that $\Omega\left(\Delta_{1}, \Delta_{2}\right)<0$ for all $\left|\Delta_{1}\right| \leq \delta_{1}$ and $\left|\Delta_{2}\right| \leq \delta_{2}$.
Finally, we consider the case where $\ln \rho_{2} / \ln \rho_{1}$ is a rational number. In this case, we can perturb $\delta_{1}$ slightly to give $\tilde{\delta}_{1}=\delta_{1}-\varepsilon_{1}$ for some very small $\varepsilon_{1}$ so that $\ln \rho_{2} / \ln \tilde{\rho}_{1}$ is irrational, where $\tilde{\rho}_{1}$ is the corresponding perturbed $\rho_{1}$. Now the proof for the irrational case can apply and we have $\Omega\left(\Delta_{1}, \Delta_{2}\right)<0$ for all $\left|\Delta_{1}\right| \leq \tilde{\delta}_{1}$ and $\left|\Delta_{2}\right| \leq \delta_{2}$. Since $\tilde{\delta}_{1}$ can be made arbitrarily close to $\delta_{1}, \Omega\left(\Delta_{1}, \Delta_{2}\right)<0$ still holds for $\left|\Delta_{1}\right| \leq \delta_{1}$ and $\left|\Delta_{2}\right| \leq \delta_{2}$ by continuity.

\section{REFERENCES}

[1] R. E. Kalman, "Nonlinear aspects of sampled-data control systems," in Proc. Symp. Nonlin. Circuit Anal., Brooklyn, NY, 1957, vol. VII, pp. 273-313.

[2] J. B. Slaughter, "Quantization errors in digital control systems," IEEE Trans. Autom. Control, vol. AC-9, no. 1, pp. 70-74, Jan. 1964.

[3] D. F. Delchamps, "Stabilizing a linear system with quantized state feedback," IEEE Trans. Autom. Control, vol. 35, no. 8, pp. 916-924, Aug. 1990.

[4] W. S. Wong and R. W. Brockett, "Systems with finite communication bandwidth constraints II: Stabilization with limited information feedback," IEEE Trans. Autom. Control, vol. 44, no. 5, pp. 1049-1053, May 1999.

[5] R. W. Brockett and D. Liberzon, "Quantized feedback stabilization of linear systems," IEEE Trans. Automat. Control, vol. 45, no. 7, pp. 1279-1289, Jul. 2000.

[6] G. N. Nair and R. J. Evans, "Stabilization with data-rate-limited feedback: Tightest attainable bounds," Syst. Control Lett., vol. 41, no. 1, pp. 49-56, 2000.

[7] N. Elia and K. Mitter, "Stabilization of linear systems with limited information," IEEE Trans. Autom. Control, vol. 46, no. 9, pp. 1384-1400, Sep. 2001.

[8] G. N. Nair and R. J. Evans, "Exponential stabilisability of finite-dimensional linear systems with limited data rate," Automatica, vol. 39, no. 4, pp. 585-593, 2003.

[9] M. Fu and L. Xie, "The sector bound approach to quantized feedback control," IEEE Trans. Autom. Control, vol. 50, no. 11, pp. 1698-1711, Nov. 2005.

[10] D. Liberzon and J. Hespanha, "Stabilization of nonlinear systems with limited information feedback," IEEE Trans. Autom. Control, vol. 50, no. 6, pp. 910-915, Jun. 2005.

[11] H. Ishii and T. Basar, "Remote control of LTI systems over networks with state quantization," Syst. Control Lett., vol. 54, pp. 15-31, 2005.

[12] G. Zhai, Y. Matsumoto, X. Chen, J. Imae, and T. Kobayashi, "Hybrid stabilization of discrete-time LTI systems with two quantized signals," Int. J. Appl. Math. Comput. Sci., vol. 15, no. 4, pp. 509-516, 2005.

[13] B. Picasso and A. Bicchi, "On the stabilization of linear systems under assigned I/O quantization," IEEE Trans. Autom. Control, vol. 52, no. 10, pp. 1994-2000, Oct. 2007.

[14] G. Gu and L. Qiu, "Networked stabilization of multi-input systems with channel resource allocation," in Proc. 17th IFAC World Congress, Seoul, Korea, Jul. 2008, pp. 625-630.

[15] C. E. de Souza, M. Fu, and L. Xie, " $\boldsymbol{H}_{\infty}$ analysis and synthesis of discrete-time systems with time-varying uncertainty," IEEE Trans. Autom. Control, vol. 38, no. 3, pp. 459-462, Mar. 1993.

[16] K. Zhou, J. C. Doyle, and K. Glover, Robust and Optimal Control. Upper Saddle River, NJ: Prentice Hall, 1996.

[17] M. C. de Oliveira, J. C. Geromel, and J. Bernussou, "Extended $\boldsymbol{H}_{2}$ and $\boldsymbol{H}_{\infty}$ norm characterizations and controller parametrizations for discrete-time systems," Int. J. Control, vol. 75, no. 9, pp. 666-679, 2002.

[18] J. Butcher, "Approximation of irrational numbers by rational numbers," New Zealand Math. Mag., vol. 36, no. 2, p. 58, 1999. 